\title{
Compliant Impedance Control for a Redundant Manipulator During Human Robot Interaction
}

\author{
Yiming Jiang ${ }^{1,2}$, Chenguang Yang ${ }^{3 *}$, Zhaojie $\mathrm{Ju}^{2}$, Andy Annamalai, ${ }^{4}$ and Honghai Liu ${ }^{2}$ \\ 1. Key Laboratory of Autonomous Systems and Networked Control, College of Automation Science and Engineering, \\ South China University of Technology, Guangzhou 510640, China. \\ 2. School of Computing, University of Portsmouth, Portsmouth PO1 3HE, UK. \\ 3. Zienkiewicz Center for Computational Engineering, Swansea University, Swansea SA1 8EN, UK. \\ 4. Moray College, University of Highlands and Islands, Elgin IV30 1JJ, UK. \\ *Corresponding author: Chenguang Yang (cyang@theiet.org)
}

\begin{abstract}
Robot control with a compliant motion behaviour is important to guarantee the safety of human robot interaction. In this paper, we aim to guarantee the desired task impedance of the end-effector and to ensure a compliant behaviour of a redundant robot during human robot interactions. A task space impedance control scheme is proposed by designing a transferred impedance error, such that the robot end-effector can be governed to follow a target impedance model. Additionally, a low-priority controller is designed using the null space projection, such that compliant joint motion is guaranteed without affecting the main task. The stability of the whole system is illustrated using a conditional Lyapunov theorem. Simulation studies based on a plane redundant robot is carried out to demonstrate the validity of the proposed controller.
\end{abstract}

Index Terms-Redundant Robot, Human-robot Interaction, Null Space Control, Impedance Control.

\section{INTRODUCTION}

The potential of co-operations between human beings and robots in a co-existent environment has elicited immense interests in industry and academia, which lead to the recent growth of research in human-robot interactions (HRI) [1][6]. The traditional industrial robots must be set in cages to avoid contacting with the surrounded people. However, in human robot interaction scenarios, the contact may easy to be happened. For example, when robots picking up a tool in a human surrounded environment, collision may easy to happen to the human. Traditional industrial robots are employed with a high stiffness controller to guarantee the motion precision. This often resulted in rigid movements which may cause serious damages during HRI. Therefore, to guarantee the interaction safety, compliant and flexible robot motion control becomes essential. In the last decade, a number of devices have been presented to improve the compliant of the robots, such as series elastic actuators [7], [8]. However, it is still challenging to design an efficient robot control scheme, especially when HRI occurs in the complex environment.

In previous studies, various control frameworks have been designed [9]-[13] to guarantee the safety human-robot collaboration. In [9], a stereo camera based shared control system was proposed for obstacle avoidance in a teleoperated task, where the robot body was able to guarantee the stability of the robot control while avoid an obstacle. In this work, the objective of the position control is to achieve a fast and accurate position or velocity tracking performance with the capability of collision avoidance, such that the robot are able to avoid the possible collision points. However, controlling a robot only to track a predefined trajectory is insufficient to ensure a safe and compliant interaction. The compliant control of robot joints will be more desirable when the robot operates in a human surrounded environment.

The impedance control was widely investigated as a robust force control method, which regulates the dynamic behaviour at the interaction point [11]-[14]. An impedance control mechanism was developed to task a desired impedance model in [12] to active control the interaction of robots. An impedance control framework was developed for robot interacting with unknown environments, in which the controller could update the robot impedance parameters in terms of the interaction performance [13]. In these control methods, although the desired impedance control behaviour was achieved in Cartesian space, only a few researchers have investigated the impedance control in the task space and joint space simultaneously. There are still limitations in achieving the compliant control of the whole robot body rather than the task space impedance control.

Motivated by the above discussion, here we design a hierarchical control framework, which could more detailed specify the control task at both the robot end-effector and at robot joints to further improve the compliant of the robot during human robot interaction. Particularly, a compliant control of a redundant manipulator with a multi-priority impedance target is designed, which enables the robot to follow two hierarchies of impedance targets based on the interaction with external environments. In order to further improve the compliant of the robot during human robot interaction, here we can design a hierarchical control, which could more detailed specify the control task at both the robot end-effector and at robot joints [15], [16]. Additionally, a task space impedance control scheme is proposed by using a transferred impedance error, such that the robot end-effector can be governed to follow a target impedance model. Moreover, a low prior controller is designed to achieve a compliant joint motion without affecting 
the main task.

The rest of the paper is organized as follows. Section II gives the preliminaries such as kinematics and dynamics of the redundant robot. In Section III, the proposed impedance control framework are detailed. The simulation results are presented in Section IV. Concluding remarks are presented in Section V.

\section{PRELIMINARIES}

\section{A. Kinematics}

The robot system studied in this paper a robot manipulator with $n$ DOF, which operated in a $m$ dimensional Cartesian Space. A redundant robot system means that the dimensions of joint space are larger than the dimensions of Cartesian space, i.e., $m<n$, and therefore we could have more joint DOF than needed to complete the task. The mapping between joint space velocity and Cartesian space velocity can be related by the equation as below

$$
\dot{x}=J(q) \dot{q}
$$

where $\dot{x}$ denotes the Cartesian space velocity, $q$ denotes the joint angle and $\dot{q}$ denotes the joint velocity, respectively. $J(q)$ denote the robot Jacobian matrix.

Conventionally, the inverse kinematics is described by the equation $\dot{q}=J^{\dagger}(q) \dot{x}$, where $J^{\dagger}$ is the well known pseudoinverse of the $J(q)$. For a redundant manipulator, however, the inverse solution of joint velocity $\dot{q}$ with respect to a given $\dot{x}$ is not unique, since we have $n$ state variable but only $m$ equalities in (1). A general inverse solution is described as below,

$$
\dot{q}=J^{\#} \dot{x}+N \dot{q}_{n}
$$

where $J^{\#}(q)$ is the generalized inverse which satisfying the relationship $J=J J^{\#} J, N$ is a null space matrix defined as $N=I-J^{\#} J$, and $\dot{q}_{n}$ is an auxiliary velocity vector.

\section{B. Joint Space Decomposition}

Then, using a joint space decomposition method, the inverse solution of the joint velocity is described as below,

$$
\dot{q}=N \dot{q}_{n}=L \vartheta
$$

where $L(q)$ is an auxiliary matrix satisfied that $J(q) L(q)=\mathbf{0}$, with $\vartheta$ being an auxiliary velocity to represent $q_{n}$. Also, the inverse solution of $\vartheta$ is given as [17]

$$
\vartheta=L(q)^{\#} \dot{q}_{n}
$$

where $L(q)^{\#}=\left(L(q)^{T} M(q) L(q)\right)^{-1} L(q)^{T} M(q)$ is the inertial-weight generalized inverse of $L(q)$.

Substituting (3) and (2), the joint velocity $\dot{q}$ can be rewritten as

$$
\dot{q}=J^{\#} \dot{x}+L \vartheta
$$

Differentiate (5) with respect to time, a general solution of $\ddot{q}$ is further described as below

$$
\ddot{q}=J^{\#}(\ddot{x}-\dot{J} \dot{q})+L\left(\dot{\vartheta}-\dot{L}^{\#} \dot{q}\right)
$$

\section{Dynamics}

Following the Lagrangian formulation, the robot dynamics of a series manipulator is formulated as follows

$$
M(q) \ddot{q}+C(q, \dot{q}) \dot{q}+G(q)+\tau_{e}=\tau
$$

where $M(q)$ is an inertia matrix, $C(q, \dot{q})$ is a Coriolis and centrifugal matrix, $G(q)$ is a gravity term, $q$ is the joint angles, $\tau$ denotes the joint torque while $\tau_{d}$ is the external torque applied on the robot joints.

In terms of the equality that $J^{T} J^{\# T}+N^{T}=I$ and considering (3) and (4), we can derive that $I=J^{T} J^{\# T}+L^{\# T} L^{T}$. Then, substituting (6) into (7), the robot dynamic model (7) can be rewritten as

$$
\begin{aligned}
\tau=\left(J^{T} J^{\# T}+L(q)^{\# T} L(q)^{T}\right)\left(M J^{\#} \ddot{x}-M J^{\#} \dot{J} \dot{q}\right. \\
\left.+M L(q) \dot{\vartheta}-M L(q) \dot{L}(q)^{\#} \dot{q}+C(q, \dot{q}) \dot{q}+g(q)+\tau_{e}\right) \\
=J^{T} J^{\# T}\left(M J^{\#} \ddot{x}-M J^{\#} \dot{J} \dot{q}+C(q, \dot{q}) \dot{q}+g(q)+\tau_{e}\right) \\
\quad+L^{\# T} L^{T}\left(M L \dot{\vartheta}-M L \dot{L}^{\#} \dot{q}+C(q, \dot{q}) \dot{q}+g(q)+\tau_{e}\right) \\
\quad+J^{T} J^{\# T}(M L(\dot{\vartheta}-\dot{q}))+L^{\# T} L^{T}\left(M J^{\#}(\ddot{x}-\dot{J} \dot{q})\right)
\end{aligned}
$$

Notice the terms on the right hand side of (8) can be divided into three groups, i.e., the task space control force, the the null space control torque, and the forces and torques which are coupled. Hence, we need to decouple the system to control the task space motion and the null space motion independently. Here, we choose the $J^{\#}$ as the inertial weight generalized inverse matrix as $J^{\#}=M^{-1} J^{T}\left(J M^{-1} J^{T}\right)^{-1}$, such that $J^{\#} M L(q)=0$ and hence we have

$$
J^{T} J^{\# T}(M L(\dot{\vartheta}-\dot{q}))=L^{\# T} L^{T}\left(M J^{\#}(\ddot{x}-\dot{J} \dot{q})\right)=0
$$

Then the system dynamic (8) can be rewritten to

$$
\begin{aligned}
\tau=J^{T} & J^{\# T}\left(M J^{\#}(\ddot{x}-\dot{J} \dot{q})+C(q, \dot{q}) \dot{q}+g(q)+\tau_{e}\right) \\
& +L^{\# T} L^{T}\left(M L\left(\dot{\vartheta}-\dot{L}^{\#} \dot{q}\right)+C(q, \dot{q}) \dot{q}+g(q)+\tau_{e}\right)
\end{aligned}
$$

Now we can see that the system (10) is decoupled and the controller can be designed for the task space motion and null space motion, respectively.

\section{Controller Design}

For a redundant robot, the joint control torque can be written to two parts, a task space control force which relate to the force applied on the end-effector, and a null space control torque with respect to the torque of the null space, which can be described as below.

$$
\tau=J^{T} \tau_{x}+N^{T} \tau_{n}
$$

where $\tau$ is the joint control torque, $\tau_{x}$ denotes the designed task space force, and $\tau_{n}$ is the null space torque specified by the designer. The overall control scheme is depicted as shown in Fig.1. 


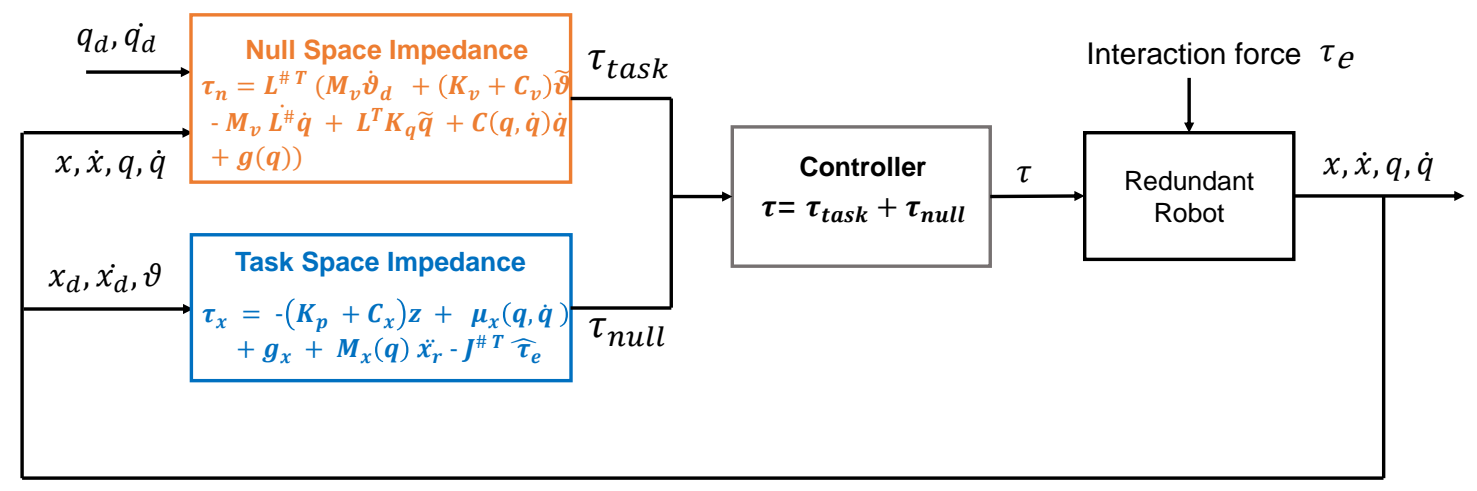

Fig. 1. Phase trajectory of the robot control

\section{A. Task space controller}

The control objective of task space motion is to govern the end-effector to follow a desired impedance model which is given by

$$
M_{d}\left(\ddot{x}-\ddot{x}_{0}\right)+C_{d}(q, \dot{q})\left(\dot{x}-\dot{x}_{0}\right)+G_{d}(q)\left(x-x_{0}\right)=-f_{e}
$$

where $M_{d}, G_{d}$ and $C_{d}$ are the desired inertia, stiffness and damping matrices, respectively, and $x_{0}$ is the position of the end-effector. $f_{e}$ is interaction force exerted on the robot endeffector. Generally, the impedance model regulates the system dynamics with respect to the interaction force and motion error, by which the robot end-effector can perform a compliant motion behaviour in the presence of an external force.

To describe the above equations in a more compact way, the following error signals can be selected as

$$
w=M_{d} \ddot{\gamma}+C_{d} \dot{\gamma}+G_{d} \gamma+f_{e}
$$

where $\gamma=x-x_{0}$. Thus, the aim of the control framework is to design a learning control law to guarantee $\lim _{t \rightarrow \infty} w(t)=0$. For ease of the control design, an equivalent impedance error is designed as follows,

$$
w_{b}=K_{f} w=\ddot{\gamma}+K_{d} \dot{\gamma}+K_{p} \gamma+K_{f} f_{e}
$$

where $K_{d}=M_{d}^{-1} C_{d}, K_{p}=M_{d}^{-1} G_{d}, K_{f}=M_{d}^{-1}$. Note that the desired impedance $M_{d}$ should be chosen as a non-singular matrix.

To facilitate the controller design, let us introduce two positive definite filtered matrices $\Upsilon$ and $\Psi$ and a filtered vector $\xi$ as

$$
\left\{\begin{array}{l}
\Upsilon+\Psi=K_{d} \\
\dot{\Upsilon}+\Psi \Upsilon=K_{p} \\
\dot{\xi}+\Psi \xi=K_{f} \tau_{f}
\end{array}\right.
$$

then (14) can be further rewritten to

$w_{b}=K_{f} w=\ddot{\gamma}+(\Upsilon+\Psi) \dot{\gamma}+(\Psi \Upsilon+\dot{\Upsilon}) \gamma+\dot{\xi}+\Psi \xi=K_{f} \tau_{f}$
By introducing the an residual error vector

$$
z=\dot{\gamma}+\Upsilon \gamma+\xi
$$

then (16) can be rewritten to a slide mode form as

$$
w_{b}=\dot{z}+\Upsilon z
$$

Now the condition $\lim _{t \rightarrow \infty} w_{b}=0$ will be true if $\lim _{t \rightarrow \infty} z=$ 0 . Thus, the impedance control objective becomes

$$
\lim _{t \rightarrow \infty} z=0
$$

In terms of (7) and (11), the motion and force of the endeffector with respect to the generalized task space force $\tau_{x}$ can be described by the following equation,

$$
\tau_{x}=M_{x}(q) \ddot{x}+\mu_{x}(q, \dot{q})+g_{x}(q)-f_{e}
$$

where $M_{x}(q)=J^{\# T} M J^{\#}, \mu_{x}(q, \dot{q})=\left(J^{\# T} C-M \dot{J}\right) \dot{q}$, $g_{x}=J^{\# T} g(q), f_{e}=J^{\# T} \tau_{e}$.

Thus, to guarantee the desired impedance motion behaviour, the task space controller can be designed as

$$
\tau_{x}=-\left(K_{p}+C_{x}\right) z+\mu_{x}(q, \dot{q})+g_{x}+M_{x}(q) \ddot{x}_{r}-J^{\# T} \hat{\tau}_{e}
$$

where $K_{p}$ is a positive parameter, $\tau_{e}$ is the measured external joint torque, and $C_{x}=\left(J^{\# T} C(q, \dot{q})-M_{x} \dot{J}\right) J^{\#}$.

By defining a reference variable $\dot{x}_{r}$ as $\dot{x}_{r}=\dot{x}_{0}-\Upsilon \gamma-\xi$ then we have $z=\dot{x}-\dot{x}_{r}$ and $\dot{z}=\ddot{x}-\ddot{x}_{r}$. Hence, the system dynamics can be rewritten as

$$
M_{x}(q) \dot{z}=\tau_{x}-\mu_{x}(q, \dot{q})-g_{x}(q)+f_{e}-M_{x}(q) \ddot{x}_{r}
$$

Using (21) and (22) yields the equation

$$
M_{x} \dot{z}=-\left(K_{p}+C_{x}\right) z
$$

\section{B. Null space controller}

By designing the velocity vector $\dot{\vartheta}$, an additional subtask can be performed by redundant joints such that the manipulator can be controlled with the desired configuration without disturbing the main task. In order to allow the robot to achieve an impedance behaviour in the configuration space, the null space control is designed such that compliant interactive motions can 
be achieved in joint space in the presence of the external forces on the robot.

Considering the system dynamics (10) and the control scheme in (11), the null space dynamics can be formulated as

$$
N^{T} \tau_{n}=L^{\# T} L^{T}\left(M L\left(\dot{\vartheta}-\dot{L}^{\#} \dot{q}\right)+C(q, \dot{q}) \dot{q}+g(q)+\tau_{e}\right)
$$

where $\tau_{e}$ is the external torque applied on the joint. The null space controller can be designed as

$$
\begin{aligned}
\tau_{n} & =L^{\# T}\left(M_{v} \dot{\vartheta}_{d}+\left(K_{v}+C_{v}\right) \tilde{\vartheta}\right. \\
& \left.-M_{v} \dot{L}^{\#} \dot{q}+L^{T} K_{q} \tilde{q}+C(q, \dot{q}) \dot{q}+g(q)\right)
\end{aligned}
$$

where $M_{v}=L^{T} M L, \vartheta_{d}$ is the desired null space velocity, $\tilde{\vartheta}=\vartheta-\vartheta_{d}, K_{q}$ and is a designed positive definite matrix and $\tilde{q}=q-q^{*}$, with $q^{*}$ being the joint configuration when the desired task impedance is achieved, $C_{v}=\left(L^{T} C(q, \dot{q})-\right.$ $\left.M_{v} \dot{L}^{\#}\right) L$ satisfying the property that $\dot{M}_{v}-2 C_{v}$ is a skewsymmetric matrix. Substituting (25) into (24) and considering that $N^{T}=L^{\# T} L^{T}$, the closed-loop dynamics of the null space system can be reformulated as

$$
L^{\# T}\left(M_{v} \dot{\tilde{\vartheta}}+\left(K_{v}+C_{v}\right) \tilde{\vartheta}+L^{T} K_{q} \tilde{q}\right)=L^{\# T} L^{T} \tau_{e}
$$

Multiplying $L^{T}$ on both side of (26), we have

$$
M_{v} \dot{\tilde{\vartheta}}+\left(K_{v}+C_{v}\right) \tilde{\vartheta}+L^{T} K_{q} \tilde{q}=L^{T} \tau_{e}
$$

Note that $L^{T} \tau_{e}$ can be interpreted as a projection of the external torque applied on null space, such that (27) represents the null space impedance equation with inertia $M_{v}$, damping $K_{v}$ and a projected torque $L^{T} K_{q} \tilde{q}$.

\section{Stability Analysis}

In this section, stability analysis of the proposed control framework will be performed based on a conditional stability.

Theorem 1: Given a redundant robot manipulator whose dynamics is described in (7), the controller (21) and (25) can guarantee a regulation of the desired impedance model in the task space while providing a compliant behaviour in the joint space, i.e., $\lim _{t \rightarrow \infty} z=0$ when the external force is accurately measured, the null space velocity error $\vartheta$ converge to zero and the boundedness of all the signals of the closed-loop system is achieved.

At first, let us considered the following Lyapunov candidate as

$$
V_{z}=\frac{1}{2} z^{T} M_{x} z
$$

The time derivative of (28) yields,

$$
\dot{V}_{z}=z^{T} M_{x} \dot{z}+\frac{1}{2} z^{T} \dot{M}_{x} z
$$

Substituting (23) into (29), we have

$$
\dot{V}_{z}=z^{T}\left(-K_{p} z-C_{x} z\right)+\frac{1}{2} z^{T} \dot{M}_{x} z
$$

Hence, we can derive that

$$
\dot{V}_{z} \leq-z^{T} K_{p} z
$$

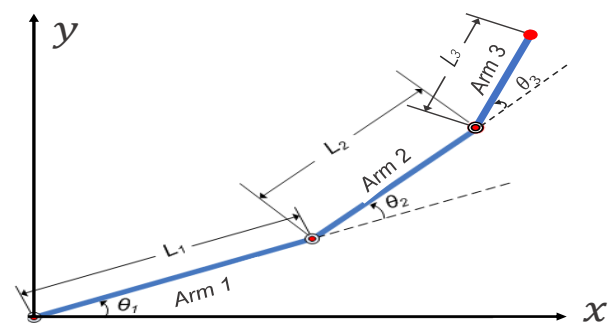

Fig. 2. The 3 DOF planner robot

TABLE I

PARAMETERS OF ROBOTIC

\begin{tabular}{llll}
\hline \hline Parameters & Link 1 & Link 2 & Link 3 \\
\hline link length $(\mathrm{m})$ & $l_{1}=1.6$ & $l_{2}=1.3$ & $l_{3}=0.7$ \\
link mass $(\mathrm{kg})$ & $m_{1}=10$ & $m_{2}=8$ & $m_{3}=2$ \\
Inertia $\left(\mathrm{kgm}^{2}\right)$ & $I_{1}=50$ & $I_{2}=50$ & $I_{3}=50$ \\
link distance $(\mathrm{m})$ & $l_{c_{1}}=0.8$ & $I_{c_{2}}=0.65$ & $l_{c_{3}}=0.35$ \\
\hline \hline
\end{tabular}

where the equality $2 z^{T} C_{x} z=z^{T} \dot{M}_{x} z$ holds. According to Lyapunov theorem, we can obtain that $z$ could converge to zero, i.e., $\lim _{t \rightarrow \infty} \dot{x} \rightarrow \dot{x}_{r}$.

Under the condition of the stability under the task space control, we will further show the stability of the whole system.

$$
V_{\vartheta}=\frac{1}{2} \tilde{\vartheta}^{T} M_{v} \tilde{\vartheta}+\frac{1}{2} \tilde{q}^{T} K_{d} \tilde{q}
$$

The time derivative of (32) can be derived as

$$
\dot{V}_{\vartheta}=\tilde{\vartheta}^{T} M_{v} \dot{\tilde{\vartheta}}+\frac{1}{2} \tilde{\vartheta}^{T} \dot{M}_{v} \tilde{\vartheta}+\dot{q}^{T} K_{d} \tilde{q}
$$

The combination of (27) and (32) yields,

$$
\begin{aligned}
& \dot{V}_{\vartheta}=\tilde{\vartheta}^{T} M_{v} \dot{\tilde{\vartheta}}+\frac{1}{2} \tilde{\vartheta}^{T} \dot{M}_{v} \tilde{\vartheta}+\dot{\tilde{q}}^{T} K_{d} \tilde{q} \\
& \leq \tilde{\vartheta}^{T}\left(-\left(K_{v}+C_{v}\right) \tilde{\vartheta}-L^{T} \tau_{e}-L^{T} K_{q} \tilde{q}\right)+\tilde{\vartheta}^{T} \dot{M}_{v} \tilde{\vartheta}+\dot{\tilde{q}}^{T} K_{d} \tilde{q} \\
& \leq \tilde{\vartheta}^{T}-\left(K_{v} \tilde{\vartheta}-L^{T} \tau_{e}-L^{T} K_{q} \tilde{q}\right)+\dot{\tilde{q}}^{T} K_{d} \tilde{q}
\end{aligned}
$$

Considering that $\vartheta^{T} L^{T} \tau_{e}=0$ and $L \tilde{\vartheta}=\dot{\tilde{q}}$ in the case that $z=0$, then we have

$$
\dot{V}_{\vartheta} \leq-\tilde{\vartheta}^{T} K_{v} \tilde{\vartheta} \leq 0
$$

Therefore, we can derive that $\tilde{\vartheta} \rightarrow 0$. Also, according to the LaSlle's invariant principle, $\tilde{q}$ is also bounded, which implies that the joints can achieve a desired configuration $q^{*}$ to minimization the external torque $\tau_{e}$.

\section{Simulation Results}

To further demonstrate the validly of the proposed control method, a 3 DOF planner robot is employed in the simulation studies as depicted in Fig. 2. Table.I gives the system parameters of the robot link. The dynamics model of the robot used in the simulation is presented as in (7) as 


$$
\begin{gathered}
M(q) \ddot{q}+C(q, \dot{q}) \dot{q}+G(q)+\tau_{d}=\tau \text { with [18] } \\
M(q)=\left[\begin{array}{ccc}
M_{11} & * & * \\
M_{21} & M_{22} & * \\
M_{31} & M_{32} & M_{33}
\end{array}\right] \\
C(q, \dot{q})=\left[\begin{array}{lll}
C_{11} & C_{12} & C_{13} \\
C_{21} & C_{22} & C_{23} \\
C_{31} & C_{32} & C_{33}
\end{array}\right] \quad G(q)=\left[\begin{array}{l}
G_{11} \\
G_{21} \\
G_{31}
\end{array}\right]
\end{gathered}
$$

In the simulations, we employ a high priority impedance controller of the end-effector, and a secondary null space control joint space to guarantee the compliant motion behaviour. The robot end-effector is controlled to track a desired Cartesian space point and then holds on to follow the desired impedance models. The end-effector position and velocity of the robot are initialized as $x(0)=[0.4,2]$ and $\dot{x}(0)=[0,0]$, respectively. The control parameters are chosen as $K_{p}=$ $\operatorname{diag}\{100,100,100\}$, and $K_{v}=\operatorname{diag}\{10,10,10\}$. The desired impedance model is chosen as $M_{d}=0.1 I, C_{D}=10 I$ and $G_{d}=0.1 I$, where $I$ denotes the unit matrix.

The control performance of our proposed impedance control is depicted as shown in Figs.3 - 5, with the interaction force chosen to be $f_{e}=[20,10]^{T}$. We can see that, the end-effector moves away from the desired point when an interaction force is added, and the stable tracking is achieved after 4 seconds. Also, the convergence of the impedance error can be observed from the subfigure of the Fig.3, which shows that the target impedance performance is achieved. The control torque in task space and null space are depicted in Fig.4 and Fig.5. We can see that all these signals are bounded. Comparison studies with different level of the interaction force have also been carried out, and simulation results are depicted in Fig.6 and Fig.7. From the figures we can see that, stable interaction performance is achieved. When the interaction force increases, the impedance error $w$ slightly increases and converges to a small neighbourhood around zero. Thus, satisfactory impedance tracking performance is ensured.

\section{CONCLUSION}

This paper develops a robot controller for a redundant manipulator to guarantee the desired task impedance of endeffector, and also to ensure a compliant behaviour of the robot joints by using the redundant degree of freedom. A task space impedance control scheme is proposed by using a transferred impedance error, such that the robot end-effector can be accurately governed to follow a target impedance model. In addition, a low priori controller is designed based on a null space projection, such that a compliant joint motion is guaranteed when external force is applied on the robot. Stability analysis based on the conditional stability has shown the stability of the overall system. Simulations based on a plane redundant robot have demonstrated the effectiveness of the proposed impedance control algorithm.

\section{ACKNOWLEDGMENT}

This work was supported in part by Engineering and Physical Sciences Research Council (EPSRC) under Grant
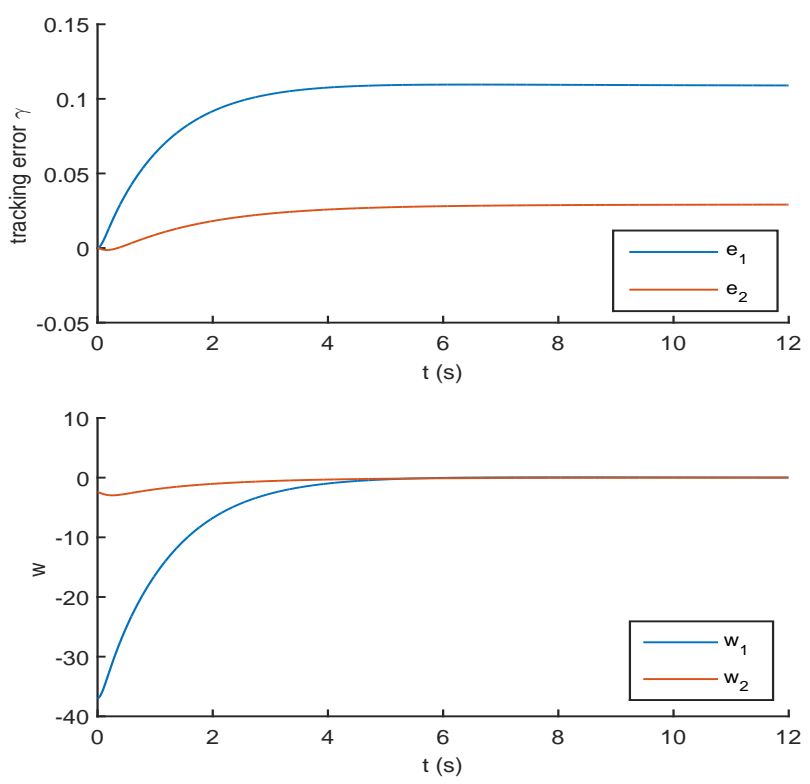

Fig. 3. Tracking error of the end-effector $e$ and impedance error $w$ with $\left(f_{e}=[20,10]^{T}\right)$

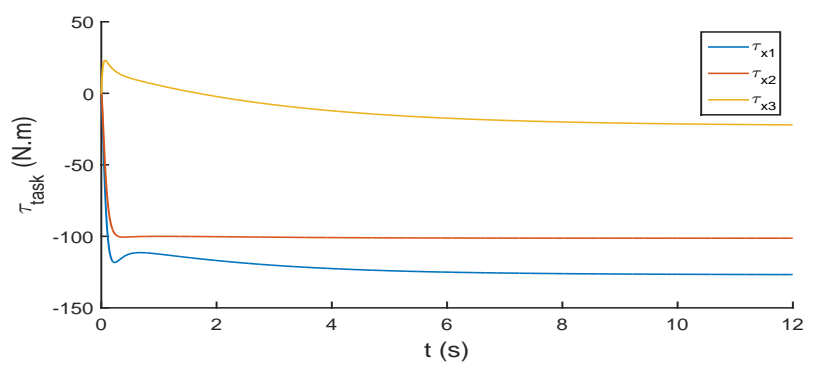

Fig. 4. Control input of the task space controller

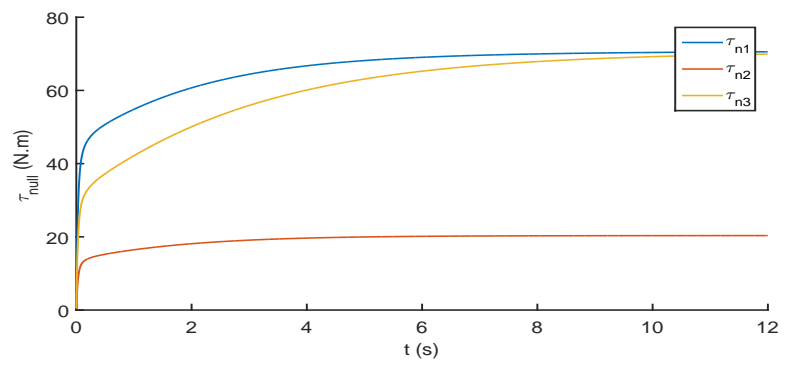

Fig. 5. Control input of the null space controller

No. EP/S001913/1, the Royal Society International Exchanges Award No. IE170247, the EU Seventh Framework Programme (FP7)-ICT under Grant No. 611391, the Nature Science Foundation of China under Grant No. 51575412, 51575338 and 51575407 and the Research Project of State Key Lab of Digital Manufacturing Equipment and Technology of China under Grant No. DMETKF2017003.

\section{REFERENCES}

[1] C. Yang, G. Ganesh, S. Haddadin, S. Parusel, A. Albu-Schaeffer, and E. Burdet, "Human-like adaptation of force and impedance in stable and 

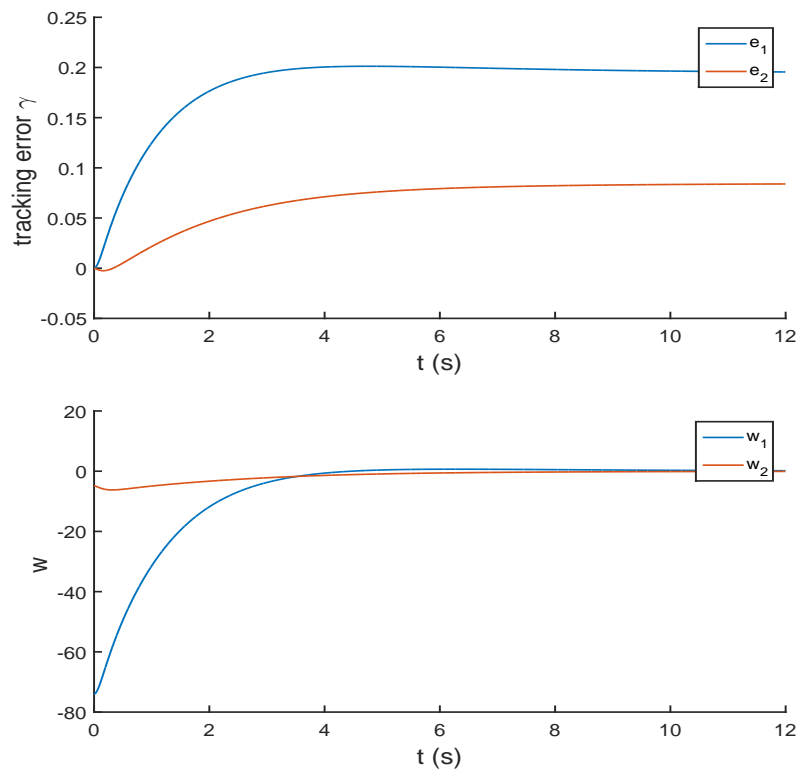

Fig. 6. Tracking error of the end-effector $e$ and impedance error $w$ with $\left(f_{e}=[50,25]^{T}\right)$
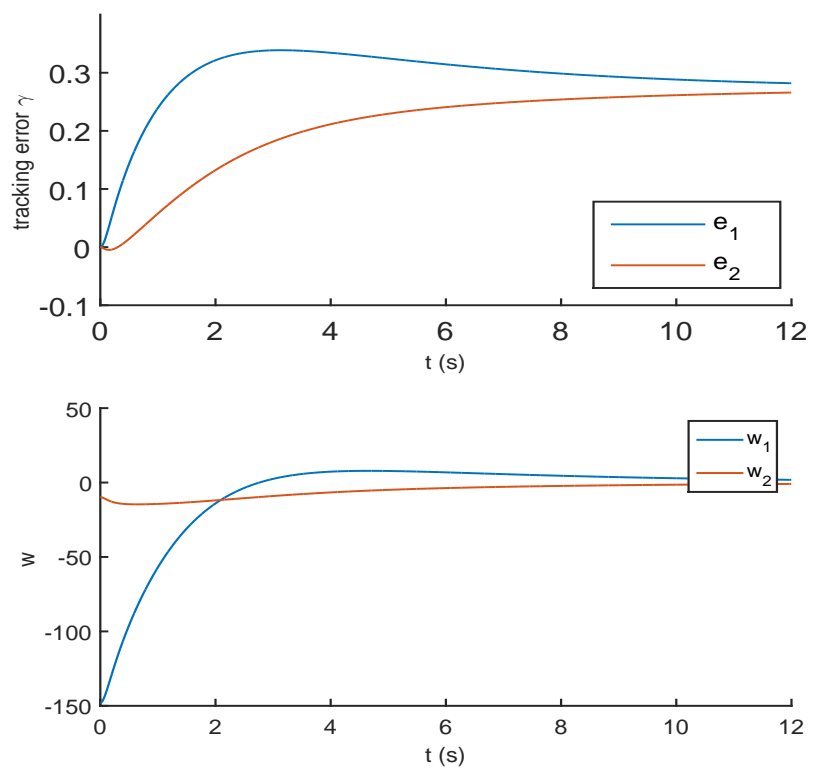

Fig. 7. Tracking error of the end-effector $e$ and impedance error $w$ with $\left(f_{e}=[100,50]^{T}\right)$

unstable interactions," IEEE transactions on robotics, vol. 27, no. 5, pp. 918-930, 2011.

[2] Y. Li, S. S. Ge, C. Yang, X. Li, and K. P. Tee, "Model-free impedance control for safe human-robot interaction," in Robotics and Automation (ICRA), 2011 IEEE International Conference on. IEEE, 2011, pp. $6021-6026$

[3] C. Yang, K. Huang, H. Cheng, Y. Li, and C.-Y. Su, "Haptic identification by elm-controlled uncertain manipulator," IEEE Transactions on Systems, Man, and Cybernetics: Systems, 2017.

[4] G. Tonietti, R. Schiavi, and A. Bicchi, "Design and control of a variable stiffness actuator for safe and fast physical human/robot interaction," in Robotics and Automation, 2005. ICRA 2005. Proceedings of the 2005 IEEE International Conference on. IEEE, 2005, pp. 526-531.

[5] K. Kong, J. Bae, and M. Tomizuka, "Control of rotary series elastic actuator for ideal force-mode actuation in human-robot interaction applications," IEEE/ASME transactions on mechatronics, vol. 14, no. 1, pp. 105-118, 2009.

[6] A. Edsinger and C. C. Kemp, "Human-robot interaction for cooperative manipulation: Handing objects to one another," in Robot and Human interactive Communication, 2007. RO-MAN 2007. The 16th IEEE International Symposium on. IEEE, 2007, pp. 1167-1172.

[7] C. Yang, H. Ma, and M. Fu, Advanced Technologies in Modern Robotic Applications. Springer, 2016.

[8] F. Ficuciello, R. Carloni, L. C. Visser, and S. Stramigioli, "Porthamiltonian modeling for soft-finger manipulation," in Intelligent Robots and Systems (IROS), 2010 IEEE/RSJ International Conference on. IEEE, 2010, pp. 4281-4286.

[9] C. Yang, Y. Jiang, Z. Li, W. He, and C.-Y. Su, "Neural control of bimanual robots with guaranteed global stability and motion precision," IEEE Transactions on Industrial Informatics, vol. 13, no. 03, pp. 1162$1171,2017$.

[10] D. Guo and Y. Zhang, "Acceleration-level inequality-based man scheme for obstacle avoidance of redundant robot manipulators," IEEE Transactions on Industrial Electronics, vol. 61, no. 12, pp. 6903-6914, Dec 2014.

[11] A. Albu-Schäffer, C. Ott, and G. Hirzinger, "A unified passivity-based control framework for position, torque and impedance control of flexible joint robots," The international journal of robotics research, vol. 26, no. 1, pp. 23-39, 2007.

[12] Y. Li, S. Sam Ge, and C. Yang, "Learning impedance control for physical robot-environment interaction," International Journal of Control, vol. 85, no. 2, pp. 182-193, 2012.

[13] Y. Li and S. S. Ge, "Impedance learning for robots interacting with unknown environments," IEEE Transactions on Control Systems Technology, vol. 22, no. 4, pp. 1422-1432, 2014.

[14] E. Gribovskaya, A. Kheddar, and A. Billard, "Motion learning and adaptive impedance for robot control during physical interaction with humans," in Robotics and Automation (ICRA), 2011 IEEE International Conference on. IEEE, 2011, pp. 4326-4332.

[15] B. Siciliano and O. Khatib, Springer handbook of robotics. Springer, 2016.

[16] H. Sadeghian, L. Villani, M. Keshmiri, and B. Siciliano, "Task-space control of robot manipulators with null-space compliance," IEEE Transactions on Robotics, vol. 30, no. 2, pp. 493-506, 2014.

[17] Y. Oh, W. Chung, and Y. Youm, "Extended impedance control of redundant manipulators based on weighted decomposition of joint space," Journal of Field Robotics, vol. 15, no. 5, pp. 231-258, 1998.

[18] C. R. Carnigan, "Adaptive tracking for complex systems using reducedorder models," in IEEE International Conference on Robotics and Automation, 1990. Proceedings, 1990, pp. 2078-2083 vol.3. 